V.-A Study of Trilinear Co-ordinates: being a Consecutive Series of Seventytwo Propositions in Transversals. By the Rev. Hugh Martin, M.A., Free Greyfriars', Edinburgh. Communicated by Professor Kelland.

(Read 20th March 1865.)

\title{
INTRODUCTORY REMARKS.
}

The following series of theorems is given as an illustration of the modern method of trilinear co-ordinates, having been wrought out after perusal of $\mathrm{Mr}$ Ferrar's very lucid and elegant treatise on that subject. The demonstrations present no difficulty, requiring nothing more complicated than the formation of determinants of two and three places. Accordingly, after exhibiting the method of proof in a few instances, I have merely given the enunciations of the remaining propositions. As the series of theorems advances the manipulation becomes, of course, a little more complicated; but the co-ordinates and co-efficients always appear in such symmetry as very greatly abbreviates the task, and guarantees its accuracy. Two, or perhaps three, of these seventy-two theorems are known mathematical truths; but that so many new consecutive propositions should be so easily found, and so easily proved, is a convincing evidence of the simplicity, fertility, and power of this new and beautiful method.

Treated according to the ancient geometry, the contents of the following pages would constitute a volume of no mean dimensions; and some of the propositions, such as those which affirm that the six points $\mathrm{P}_{1}, \mathbf{P}_{2}, \mathbf{P}_{3}, \mathbf{P}_{4}, \mathbf{P}_{5}, \mathbf{P}_{6}$ range in a straight line, and that the seven straight lines $U_{1} U_{2} ; R_{1} R_{2}, R_{3} R_{4} ; S_{1} S_{2}, S_{3} S_{4}$; $Q_{1} Q_{3}, Q_{4} Q_{5}, Q_{2} Q_{6}$ all meet in a point, would probably have been undiscoverable.

In the admirable treatises of MULCAHY and TownsEND a few analogous propositions are demonstrated geometrically. Mr Townsend, in particular, has a chapter in his first volume, on concurrent lines and co-linear points, which falls in very closely with the kind of propositions which the following series embraces. His second volume I have not been fortunate enough to see; but the subject is only ripening for a systematic gathering-up of the propositions that have been discovered in this line of investigation, and the following pages are presented as a humble contribution towards that desirable result.

A word or two may be permitted in reference to the additions to the terminology which must be made, and generally sanctioned by mathematicians, ere such systematic digest can be successfully accomplished. I have ventured-of course only provisionally-on one or two such additions. When the co-ordinates of two points are respectively the algebraical inverses of each other, I have called these points, in reference to each other, "inverse points ;" and it is evident that a very 
fine vein of mathematical truth opens up in reference to them, which it needs only a little ingenuity to work advantageously. Thus, at a glance, it is evident that if a point moves in the straight line $l \alpha+m \beta+n \gamma=0$, its inverse moves in the locus, $\frac{l}{\alpha}+\frac{m}{\beta}+\frac{n}{\gamma}=0$; which is a conic passing through the angular points of the triangle of reference. Since writing the following pages I find $\mathrm{Mr}$ Townsens) has a chapter entitled "Theory of inverse points with respect to a circle;" and although not treated according to the trilinear method, these points, so called, will be found, I rather think, if so treated, to present only a case of what I have called "inverse points" in general. In the same manner I have called two lines "inverse" with respect to each other when the co-efficients of the co-ordinates are respectively the algebraical inverses of each other.

There is another relation between a special point and line which $I$ have not ventured to designate, but to which I would respectfully call attention as requiring designation. When lines from the angular points of a triangle are drawn through any point to intersect the opposite sides, the intersections constitute the angular points of an inscribed triangle, whose sides are known to meet the corresponding sides of the original triangle in points which range in a straight line. Instead of giving a particular designation to this line, I have used the general functional symbol; and, as its position depends exclusively on the point - say $\mathrm{P}$, I have called the line $\phi(\mathrm{P})$, in a few theorems in reference to it (Theorems XXXI.-XXXV.). Of course the inverse functional symbol $\phi^{-1}$ indicates the point in reference to the line, as the direct symbol indicates the line with reference to the point. This point and line are, indeed, with respect to each other, a species of pole and polar,- the line being the ordinary polar, not of the point but of its inverse,- - to the imaginary conic $\alpha^{2}+\beta^{2}+\gamma^{2}=0$. Manifestly a special designation is necessary in a case like this, in order to secure that ease of reference and that brevity of treatment without which the pioneering work of farther investigation is brought to a stand.

Theorem LXVI. is the prize question of the "Gentleman's Diary" for 1841; and some long but good geometrical demonstrations of it are given. The proof is perfectly simple according to the trilinear method, and the co-ordinates of the point appear in a form so elegant that one could not help seeing that it must have some singular relations and be worthy of a name. I have accordingly ventured to call it the Anapole of the two given points; and, connecting it with some of the preceding results, I find a few propositions easily deducible, such as that the anapole of two inverse points and the line joining them are pole and polar, to the imaginary conic, $\alpha^{2}+\beta^{2}+\gamma^{2}=0$. For the three concluding theorems I am indebted to a young mathematical friend-destined, I believe and trust, to scientific eminence- $\mathrm{Mr}$ GEORGE M. SMith, student in the Aberdeen University. On proposing to him the problems of finding the locus of the anapole of a central body and its planet, and 
the locus of the anapole of two points which should move away from each other in a straight line with uniform velocities, I was delighted to receive demonstrations, perfectly simple and elegant, to the effect that, in the former case, the anapole moves in a straight line, let the planet move as it may; and that, in the latter case, the locus of the anapole is a conic section, and becomes a straight line if the uniform velocities are equal; and farther, that the anapole of any two points in an ellipse circumscribing the triangle of reference is invariable. Geometers, $I$ am sure, will admire these theorems of a rising young mathematician, and will recognise the vein thus struck as promising to be a fertile one. Mr Surtu added another very beautiful property of the anapole, which turned out, on investigation, to be identical with Theorem LXV. of the following series.

Instead of defining a point by the equations $\frac{\alpha}{l}=\frac{\beta}{m}=\frac{\gamma}{n}$, we shall say the point is-- $(l, m, n)$. Instead of defining a straight line by the equation $l \alpha+m \beta+n \gamma=0$, we shall say the line is- $(l, m, n)$.

The straight line joining the points $\left(l_{1}, m_{1}, n_{1}\right),\left(l_{2}, m_{2}, n_{2}\right)$ is;-

$$
\left|\begin{array}{ll}
m_{1}, & n_{1} \\
m_{2}, & n_{2}
\end{array}\right|, \quad\left|\begin{array}{ll}
n_{1}, & l_{1} \\
n_{2}, & l_{2}
\end{array}\right|, \quad\left|\begin{array}{ll}
l_{1}, & m_{1} \\
l_{2}, & m_{2}
\end{array}\right| .
$$

The intersection of the two straight lines $\left(l_{1}, m_{1}, n_{1}\right),\left(l_{2}, m_{2}, n_{2}\right)$ is defined by the same expression. This identity of form is, in reality, the earliest germ of the doctrine of pole and polar; and gives rise to what is usually regarded as the first promise of that doctrine, namely, the identity of the condition that the three points $\left(l_{1}, m_{1}, n_{1}\right),\left(l_{2}, m_{2}, n_{2}\right),\left(l_{3}, m_{3}, n_{3}\right)$ shall range in a straight line, with the condition that the three straight lines $\left(l_{1}, m_{1}, n_{1}\right),\left(l_{2}, m_{2}, n_{2}\right),\left(l_{3}, m_{3}, n_{3}\right)$ shall intersect in a point; which is, in both cases,

$$
\left|\begin{array}{lll}
l_{1}, & m_{1}, & n_{1} \\
l_{2}, & m_{2}, & n_{2} \\
l_{3}, & m_{3}, & n_{3}
\end{array}\right| \equiv 0 .
$$

\section{T H E O R E M S.}

\section{THeorem 1.}

On the sides of the triangle $\mathrm{A} \mathrm{BC}$, as bases, are constructed three triangles, $\mathrm{A}_{1} \mathrm{BC}, \mathrm{AB}_{1} \mathrm{C}, \mathrm{ABC}$, similar to each other, and so placed that the angles $\mathrm{A}_{1} \mathrm{BC}=\mathrm{A} \mathrm{B} \mathrm{C}=\mathrm{A} \mathrm{B} \mathrm{C} \mathrm{C}_{1} ; \mathrm{B}_{1} \mathrm{CA}=\mathrm{B} \mathrm{C}_{1} \mathrm{~A}=\mathrm{B} \mathrm{C} \mathrm{A}$; and $\mathrm{C}_{1} \mathrm{~A} \mathrm{~B}=\mathrm{C} \mathrm{A}_{1} \mathrm{~B}=\mathrm{CA} \mathrm{B}_{1}$. Then $\mathrm{A} \mathrm{A}_{1}, \mathrm{~B} \mathrm{~B}_{1}, \mathrm{CC}_{1}$ meet in a point.

The sides of $\mathrm{ABC}$, taken as the triangle of reference, being $a, b, c$, the perpendiculars from $A_{1}$ on $a, b, c$ are, respectively,

$$
\frac{a \cdot \sin \mathrm{B}_{1} \cdot \sin \mathrm{C}_{1}}{\sin \mathrm{A}_{1}}, \frac{a \cdot \sin \mathrm{B}_{1} \cdot \sin \left(\mathrm{C}+\mathrm{C}_{1}\right)}{\sin \mathrm{A}_{1}}, \frac{a \cdot \sin \mathrm{C}_{1} \cdot \sin \left(\mathrm{B}+\mathrm{B}_{1}\right)}{\sin \mathrm{A}_{1}} \text {. }
$$


Hence the trilinear co-ordinates of $A_{1}$ are

$$
-1, \quad \frac{\sin \left(C+C_{1}\right)}{\sin C_{1}}, \frac{\sin \left(B+B_{1}\right)}{\sin B_{1}}
$$

$$
\begin{array}{lllll}
" & \text { of } B_{1} \text { are } & \frac{\sin \left(C+C_{1}\right)}{\sin C_{1}}, & -1, & \frac{\sin \left(A+A_{1}\right)}{\sin A_{1}} . \\
& =\quad \text { of } C_{1} \text { are } & \frac{\sin \left(B+B_{1}\right)}{\sin B_{1}}, \frac{\sin \left(C+C_{1}\right)}{\sin C_{1}}, & -1 .
\end{array}
$$

$$
\text { For } \frac{\sin \left(A+A_{1}\right)}{\sin A_{1}}, \frac{\sin \left(B+B_{1}\right)}{\sin B_{1}}, \frac{\sin \left(C+C_{1}\right)}{\sin C_{1}}, \text { put } f, g, h \text {. Then ;- }
$$

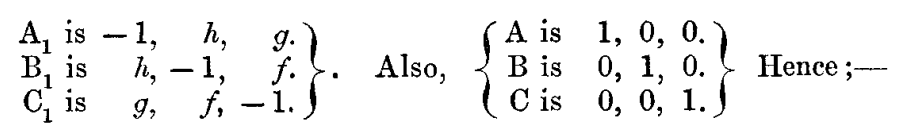

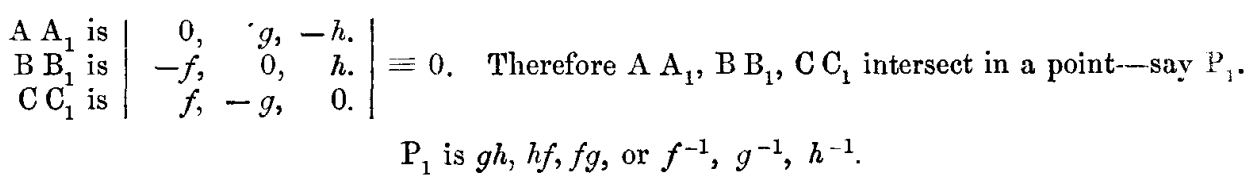

\section{Theorem 2.}

$$
\left.\begin{array}{lll}
\mathrm{B}_{1} \mathrm{C}_{1} \text { is } 1-f^{2}, & h+f g, & g+h f \\
\mathrm{C}_{1} \mathrm{~A}_{1} \text { is } h+f g, & 1-g^{2}, & f+g h \\
\mathrm{~A}_{1} \mathrm{~B}_{1} \text { is } g+h f, & f+g h, & 1-h^{2}
\end{array}\right\} . \text { Also, }\left\{\begin{array}{ll}
\mathrm{BC} \text { is } 1,0,0 . \\
\mathrm{CA} \text { is } 0,1,0 . \\
\mathrm{A} \mathrm{B} \text { is } 0,0,1 .
\end{array}\right\} \text { Hence;- }
$$

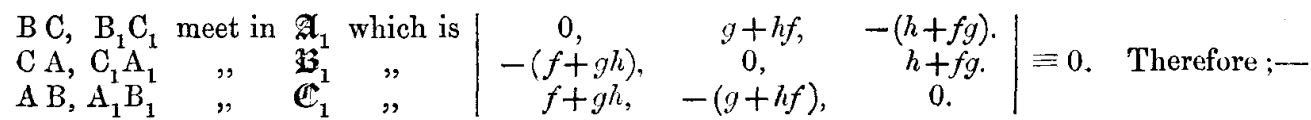

$$
\mathfrak{A}_{1} \mathfrak{\mathfrak { B }}_{1} \mathfrak{C}_{1} \text { is a straight line, viz., }(f+g h)^{-1},(g+\hbar f)^{-1},(h+f g)^{-1} \text {. }
$$

\section{Theorem 3.}

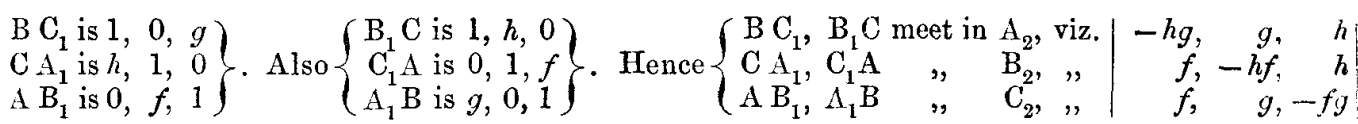


$\mathrm{P}_{2}$ is $f, g, h$.

$\mathrm{P}_{1}$ and $\mathrm{P}_{2}$ may be called inverse or reciprocal points.

\section{THEOREM 4.}

$$
\begin{array}{r|ccc}
\mathrm{BC} \text { is } & 1, & 0, & 0 . \\
\mathrm{B}_{1} \mathrm{C}_{1} \text { is } & 1-f^{2}, & h+f g, & g+h f . \\
\mathrm{B}_{2} \mathrm{C}_{2} \text { is } & -g h\left(1-f^{2}\right), & f(h+f g), & f(g+h f) .
\end{array} \mid \equiv 0 . \quad \text { Therefore;- }
$$

$\mathrm{BC}, \mathrm{B}_{1} \mathrm{C}_{1}, \mathrm{~B}_{2} \mathrm{C}_{2}$ intersect in $\mathfrak{A}_{1}$.

* $\mathrm{C} \Lambda, \mathrm{C}_{1} \Lambda_{1}, \mathrm{C}_{2} \Lambda_{2} \quad, \quad \mathfrak{B}_{1}$. $A B, A_{1} B_{1}, A_{2} B_{2} \quad " \quad \mathbb{C}_{1}$. 


\section{Theorem 5.}

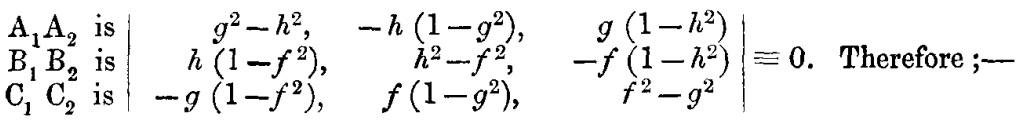

$$
\begin{aligned}
& \mathrm{A}_{1} \mathrm{~A}_{2}, \mathrm{~B}_{1} \mathrm{~B}_{2}, \mathrm{C}_{1} \mathrm{C}_{2} \text { intersect in a point,--say } \mathrm{P}_{8} \text {, viz., } f-g h, g-h f, h-f g \text {. }
\end{aligned}
$$

\section{TheOREM 6.}

$$
\begin{gathered}
\begin{array}{ccc}
\mathrm{P}_{\mathrm{I}} \text { is } \\
\mathrm{P}_{2} \text { is } \\
\mathrm{P}_{3} \text { is }
\end{array}\left|\begin{array}{ccc}
g h, & h f, & f g . \\
f-g h, & g-h f, & h-f g .
\end{array}\right| \equiv 0 . \quad \text { Therefore } \mathrm{P}_{1}, \mathrm{P}_{2}, \mathrm{P}_{3} \text { range in a straight line. } \\
\mathrm{P}_{1} \mathrm{P}_{2} \mathrm{P}_{3} \text { is } f\left(g^{2}-h^{2}\right), g\left(h^{2}-f^{2}\right), h\left(f^{2}-g^{2}\right) .
\end{gathered}
$$

Theorem 7.

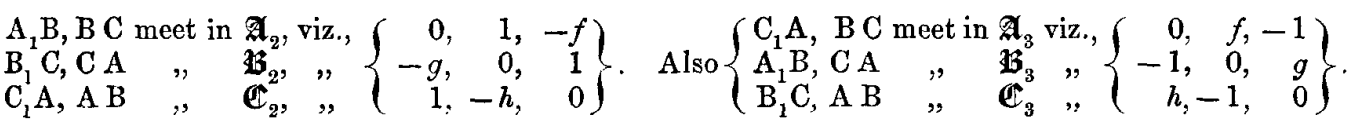

Hence ;-

$$
\left.\left.\begin{array}{ll}
\mathfrak{b}_{2} \mathfrak{C}_{2} & \text { is } \\
\mathfrak{C}_{2} \mathfrak{A}_{2} & \text { is }
\end{array}\left\{\begin{array}{ccc}
h, & 1, g h \\
h f, & f, & 1 \\
\mathfrak{A}_{2} \mathfrak{B}_{2} & \text { is }
\end{array}\right\} ; \begin{array}{ll}
\mathfrak{b}_{3} \mathfrak{C}_{3} & \text { is } \\
\mathfrak{C}_{\mathbf{3}} \mathfrak{A}_{3} & \text { is }
\end{array}\right\} \begin{array}{ccc}
g, & g h, & 1, \\
1, & h, & h f, \\
f g, & 1, & f,
\end{array}\right\}
$$

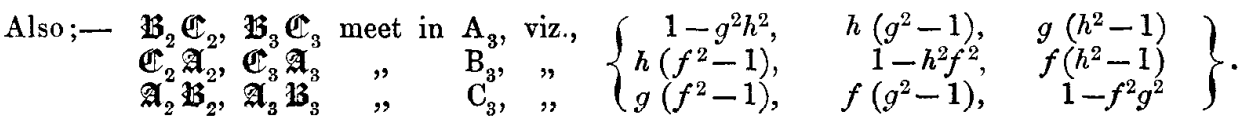

Farther ;-

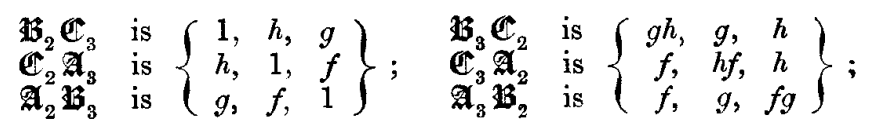

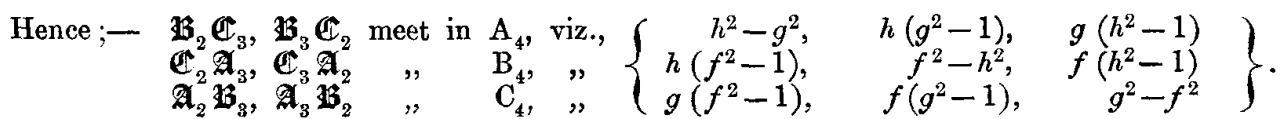

Now;-

$$
\begin{array}{l|ccc}
\text { A being } & 1, & 0, & 0 \\
\mathrm{~A}_{3} " & 1-g^{2} h^{2}, & h\left(g^{2}-1\right), & g\left(h^{2}-1\right) \\
\mathbf{A}_{4} \quad " & h^{2}-g^{2}, & h\left(g^{2}-1\right), & g\left(h^{2}-1\right)
\end{array} \mid \equiv 0 . \quad \text { Therefore;- }
$$

$\mathrm{A} \mathrm{A}_{3} \mathrm{~A}_{4}$ is a straight line.



\section{Theorem 8.}

\begin{tabular}{l|ccc}
$\mathrm{A} \mathrm{A}_{3} \mathrm{~A}_{4}$ is & 0, & $g\left(1-h^{2}\right)$, & $-h\left(1-g^{2}\right)$ \\
$\mathrm{B} \mathrm{B}_{3} \mathrm{~B}_{4}$ is & $-f\left(1-h^{2}\right)$, & 0, & $h\left(1-f^{2}\right)$ \\
$\mathrm{C} \mathrm{C}_{3} \mathrm{C}_{4}$ is & $f\left(1-g^{2}\right)$, & $-g\left(1-f^{2}\right)$, & 0
\end{tabular} \mid$\equiv 0 . \quad$ Therefore;-

$$
\mathrm{A} \mathrm{A}_{3} \mathrm{~A}_{4}, \mathrm{~B} \mathrm{~B}_{3} \mathrm{~B}_{4}, \mathrm{C} \mathrm{C}_{3} \mathrm{C}_{4} \text { meet in a point } \mathrm{P}_{4} \text {, viz., } \frac{1-f^{2}}{f}, \frac{1-g^{2}}{g}, \frac{1-h^{2}}{h} \text {. }
$$


Theorem 9.

\begin{tabular}{l|ccc}
$\mathbf{A}_{1}$ is & -1, & $h$, & $g$ \\
$\mathbf{A}_{2}$ is & $-g h$, & $g$, & $h$ \\
$\mathbf{A}_{3}$ is & $1-g^{2} h^{2}$, & $h\left(g^{2}-1\right)$, & $g\left(h^{2}-1\right)$
\end{tabular} \mid$\equiv 0 . \quad$ Therefore;-

$A_{1} A_{2} A_{3}$ is a straight line.

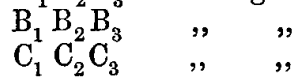

\section{Theorem 10.}

$\mathrm{B}_{3} \mathrm{C}_{3}$ is $1+f g h, \quad h+f g, \quad g+h f$; meeting $\mathbf{B} \mathrm{C}$ in $\left.\mathbf{A}_{1}\right)$

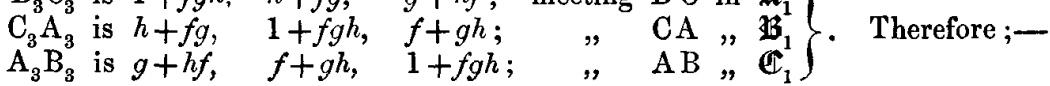

$\mathrm{B} \mathrm{C}, \mathrm{B}_{1} \mathrm{C}_{1}, \mathrm{~B}_{2} \mathrm{C}_{2}, \mathrm{~B}_{3} \mathrm{C}_{3}$ meet in $\mathfrak{A}_{1}$.

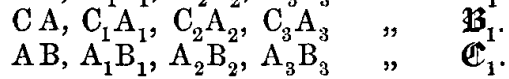

\section{Theorem 11.}



Therefore $\mathfrak{A}_{4} \mathfrak{B}_{4} \mathfrak{C}_{4}$ is a straight line, viz., $\frac{1}{(g+h f) \cdot(h-f g)}, \frac{1}{(h+f g) \cdot(f-g h)}, \frac{1}{(f+g h) \cdot(g-h f)}$

Theorem 12.



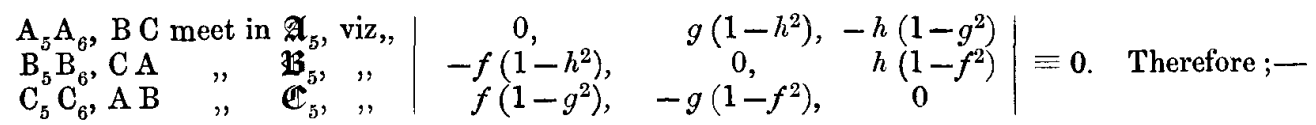

$\mathfrak{A}_{5} \mathfrak{B}_{5} \mathfrak{C}_{5}$ is a straight line, viz., $\frac{1-f^{2}}{f}, \frac{1-g^{2}}{g}, \frac{1-h^{2}}{h}$.

Theorem 13.

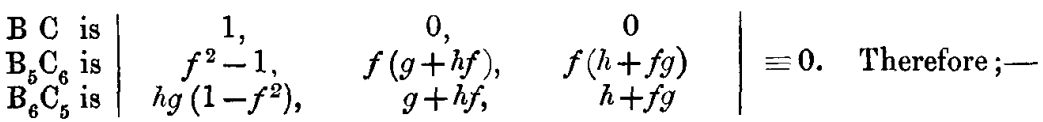

$\mathrm{B} \mathrm{C}, \quad \mathrm{B}_{5} \mathrm{C}_{6}, \quad \mathrm{~B}_{6} \mathrm{C}_{5}$ meet in a point: $\mathbf{A}_{6}$

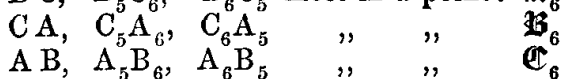


Theorem 14.

$$
\begin{gathered}
\begin{array}{c}
\mathfrak{A}_{6} \text { is } \\
\mathfrak{W}_{6}^{6} \text { is } \\
\mathfrak{C}_{6}^{6} \text { is }
\end{array}\left|\begin{array}{ccc}
0, & h+f g, & -(g+h f) \\
-(h+f g), & 0, & f+g h \\
g+h f, & -(f+g h), & 0
\end{array}\right| \equiv 0 . \quad \text { Therefore;- } \\
\mathfrak{A}_{6} \mathbf{B}_{6} \mathfrak{C}_{6} \text { is a straight line, viz., } f+g h, g+h f, h+f g . \\
\mathfrak{a}_{1} \mathfrak{B}_{1} \mathfrak{C}_{1} \text { and } \mathfrak{A}_{6} \mathfrak{B}_{6} \mathfrak{C}_{6} \text { are inverse or reciprocal lines. }
\end{gathered}
$$

\section{THEOREM 15.}

\begin{tabular}{c|ccc|cc}
$\mathfrak{B}_{1} \mathfrak{C}_{6}$ is & $(h+f g) \cdot(f+g h)$, & $(g+h f) \cdot(h+f g)$, & $(f+g h)^{2}$ & and meets B C in $\mathfrak{A}_{7}$ \\
$\mathfrak{C}_{1} \mathfrak{A}_{6}$ is & $(g+h f)^{2}$, & $(f+g h) \cdot(g+h f)$, & $(h+f g) \cdot(f+g h)$ &, & $\mathbf{C ~ A ~ i n ~} \mathfrak{B}_{7}$ \\
$\mathfrak{A}_{1} \mathbf{B}_{6}$ is & $(f+g h) \cdot(g+h f)$, & $(h+f g)^{2}$, & $(g+h f) \cdot(h+f g)$ &, & $\mathbf{A ~ B ~ i n ~} \mathfrak{C}_{7}$
\end{tabular}

$$
\begin{array}{c|cc|c}
\mathfrak{A}_{7} \text { is } & 0, & (f+g h)^{2}, & -(g+h f) \cdot(h+f g) \\
\mathfrak{B}_{7} \text { is } & -(h+f g) \cdot(f+g h), & 0, & (g+h f)^{2} \\
\mathfrak{C}_{7}^{7} \text { is } & (h+f g)^{2}, & -(f+g h) \cdot(g+h f), & 0
\end{array} \mid \equiv 0 . \quad \text { Therefore;-- }
$$

$\mathfrak{A}_{7} \mathfrak{B}_{7} \mathfrak{C}_{7}$ is a straight line, viz., $\frac{g+h f}{h+f g}, \frac{h+f g}{f+g \hat{h}}, \frac{f+g h}{g+h f}$.

\section{Theorem 16.}

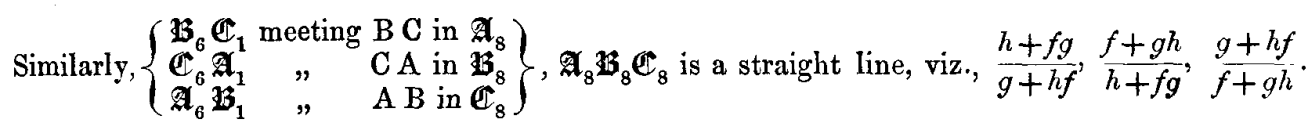

$\mathfrak{A}_{7} \mathfrak{B}_{7} \mathfrak{C}_{7}$ and $\mathfrak{A}_{8} \mathfrak{B}_{8} \mathfrak{C}_{8}$ are reciprocal lines.

\section{Theorem 17.}

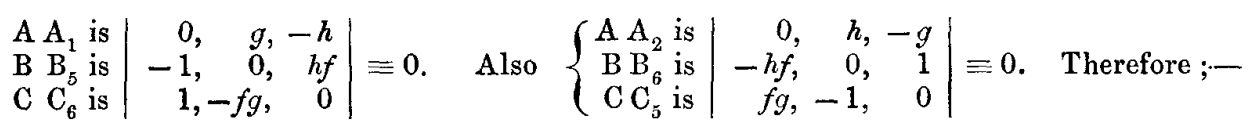

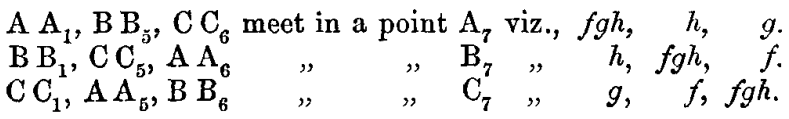

\section{Theorem 18.}

$$
\begin{aligned}
& \mathrm{A} \mathrm{A}_{2}, \mathrm{~B} \mathrm{~B}_{6}, \mathrm{CC}_{5} \text { meet in a point } \mathrm{A}_{8} \text { viz., } 1, f g, h f \text {. }
\end{aligned}
$$

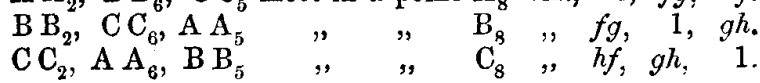

But instead of continuing the manipulation, we shall gather up these results, and continue the series of propositions. 


\section{THEOREM I.}

$A A_{1}, B B_{1}, C C_{1}$ intersect, in a point, $P_{1}$.

THEOREM II.

Let $\mathrm{B} \mathrm{C}, \mathrm{B}_{1} \mathrm{C}_{1}$ meet in $\mathfrak{A}_{1}$

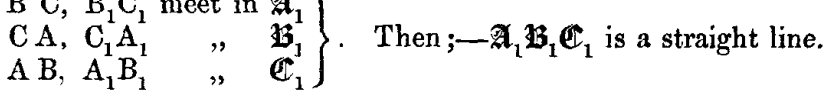

\section{ThEOREM III.}

Let $\mathrm{B} \mathrm{C}_{1}, \mathrm{~B}_{1} \mathrm{C}$ meet in $\mathrm{A}_{2}$ )



$$
\begin{aligned}
& P_{1} \text { and } P_{2} \text { are reciprocal points. }
\end{aligned}
$$

\section{Theorem IV.}

$\mathrm{B} C, \mathrm{~B}_{1} \mathrm{C}_{1}, \mathrm{~B}_{2} \mathrm{C}_{2}$ intersect in a point, and that point is $\mathfrak{A}_{1}$

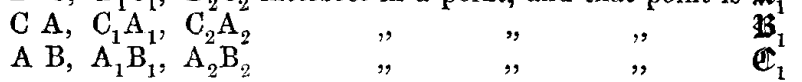

\section{THeOREM $V$.}

$$
A_{1} A_{2}, B_{1} B_{2}, C_{1} C_{2} \text { intersect in a point, } P_{3} \text {. }
$$

Theorem VI.

$\mathrm{P}_{1} \mathrm{P}_{2} \mathrm{P}_{3}$ is a straight line.

\section{Theorem VII.}

Let $\mathrm{A}_{1} \mathrm{~B}, \mathrm{BC}$ meet in $\boldsymbol{\mathfrak { A }}_{2}$

$$
\left.\begin{array}{lll}
\mathrm{B}_{1} \mathrm{C}, \mathrm{CA} & , & \mathfrak{\mathfrak { B }}_{2}^{2} \\
\mathrm{C} \mathrm{A}, \mathrm{AB} & " & \mathfrak{C}_{2}
\end{array}\right\}
$$

Also let $\mathfrak{B}_{2} \mathfrak{C}_{2}, \mathfrak{B}_{3} \mathfrak{C}_{3}$ meet in $\left.\mathrm{A}_{3}\right)$ And let $\mathfrak{B}_{2} \mathfrak{C}_{3}, \mathfrak{B}_{3} \mathfrak{C}_{2}$ meet in $\mathrm{A}_{4}$

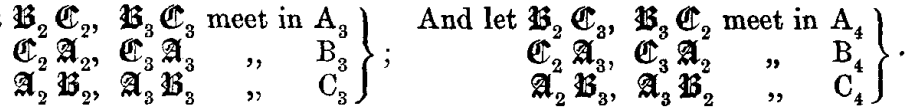

$$
\begin{aligned}
& \text { Then,- }-\mathrm{A} \mathrm{A}_{3} \mathrm{~A}_{4} \text { is a straight line. }
\end{aligned}
$$

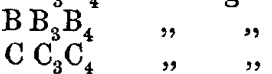

\section{THEOREM VIII.}

$A A_{3} A_{4}, B_{3} B_{4}, C C_{3} C_{4}$ meet in a point, $P_{4}$.

\section{THEOREM IX.}

$A_{1} A_{2} A_{3}$ is a straight line.

$$
\begin{array}{ll}
\mathrm{B}_{1} \mathrm{~B}_{2} \mathrm{~B}_{3} & , \quad, ",
\end{array}
$$


ThEOREM X.

$\mathrm{B} \mathrm{C}, \mathrm{B}_{1} \mathrm{C}_{1}, \mathrm{~B}_{2} \mathrm{C}_{2}, \mathrm{~B}_{3} \mathrm{C}_{3}$ all meet in $\mathfrak{A}_{1}$.

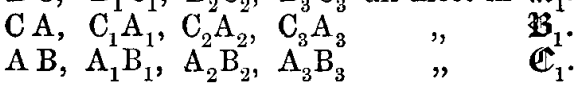

THEOREM XI.

Let $\mathrm{B} \mathrm{C}, \mathrm{B}_{4} \mathrm{C}_{4}$ meet in $\mathfrak{A}_{4}$

$\left.\begin{array}{lll}\mathrm{C} \mathrm{A}, \mathrm{C}_{4} \mathrm{~A}_{4} & \Rightarrow & \mathfrak{B}_{4}^{4} \\ \mathrm{~A} \mathrm{~B}, \mathrm{~A}_{4} \mathrm{~B}_{4} & , \quad & \mathfrak{C}_{4}^{4}\end{array}\right\}$ Then $;-\mathfrak{A}_{4} \mathfrak{B}_{4} \mathfrak{C}_{4}$ is a straight line.

THeOREM XII.

Let $\mathrm{B}_{1} \mathrm{C}, \mathrm{A}_{1} \mathrm{~B}$ meet in $\mathrm{A}_{5}$

$$
\left.\begin{array}{lll}
\mathrm{C}_{1} \mathrm{~A}, \mathrm{~B}_{1} \mathrm{C} & \Rightarrow & \mathrm{B}_{5} \\
\mathrm{~A}_{1} \mathrm{~B}, \mathrm{C}_{1} \mathrm{~A} & \Rightarrow & \mathrm{C}_{5}
\end{array}\right\}
$$

Also let $\mathrm{B} \mathrm{C}, \mathrm{A}_{5} \mathrm{~A}_{6}$ meet in $\mathfrak{A}_{5}$
And let $\left.\begin{array}{llll}\mathrm{B} \mathrm{C}_{1}, & \mathrm{C} \mathrm{A}_{1} & \text { meet in } & \mathrm{A}_{6} \\ \mathrm{CA}_{1}, & \mathrm{~A} \mathrm{~B}_{1} & , & \mathrm{~B}_{6} \\ \mathrm{~A} \mathrm{~B}_{1}, & \mathrm{~B} \mathrm{C}_{1} \quad, & \mathrm{C}_{6}\end{array}\right\}$

$\left.\begin{array}{lll}\mathrm{C} A, \mathrm{~B}_{5} \mathrm{~B}_{6} & , & \mathfrak{2}_{5}^{5} \\ \mathrm{~A} \mathrm{~B}, \mathrm{C}_{5} \mathrm{C}_{6} & , & \mathfrak{C}_{5}^{5}\end{array}\right\}$. Then $-\mathfrak{A}_{5} \mathfrak{B}_{5} \mathfrak{C}_{5}$ is a straight line.

THEOREM XIII.

$\mathrm{B} \mathrm{C}, \mathrm{B}_{5} \mathrm{C}_{6}, \mathrm{~B}_{6} \mathrm{C}_{5}$ meet in a point, $\mathfrak{A}_{6}$.

$\mathbf{C} \hat{A}, \mathrm{C}_{5} \mathrm{~A}_{6}^{6}, \mathrm{C}_{6}^{6} \mathrm{~A}_{5} \quad, \quad " \mathfrak{B}_{6}$.

$\mathrm{A} \mathrm{B}, \mathrm{A}_{5}^{5} \mathrm{~B}_{6}, \mathrm{~A}_{6}^{6} \mathrm{~B}_{5} \quad " \quad " \quad \mathbb{C}_{6}^{6}$.

THEOREM XIV.

$\mathfrak{A}_{6} \mathfrak{B}_{6} \mathfrak{C}_{6}$ is a straight line; reciprocal to $\mathfrak{A}_{1} \mathfrak{B}_{1} \mathfrak{C}_{1}$.

THEOREM XV.

Let $\mathrm{B} \mathrm{C}, \mathfrak{B}_{1} \mathfrak{C}_{6}$ meet in $\mathfrak{A}_{7}$

$\mathrm{CA}, \mathfrak{C}_{1}^{1} \mathfrak{A}_{6}^{6}$

A B, $\mathfrak{A}_{1} \mathfrak{A}_{6}^{6}$

$\left.\begin{array}{l}\mathfrak{A}_{7} \\ \mathfrak{C}_{7}^{7}\end{array}\right\}$. Then $;-\mathfrak{A}_{7} \mathfrak{B}_{7} \mathfrak{C}_{7}$ is a straight line.

THEOREM XVI.

Let $\mathrm{B} \mathrm{C}, \mathfrak{B}_{6} \mathfrak{C}_{\mathbf{1}}$ meet in $\left.\mathfrak{A}_{8}\right)$

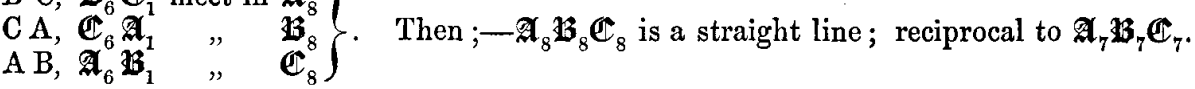

THeOREM XVII.

$\mathrm{A} \mathrm{A}_{1}, \mathrm{~B} \mathrm{~B}_{5}, \mathrm{C} \mathrm{C}_{6}$ meet in a point, $\mathrm{A}_{7}$.

$\begin{array}{lllll}\mathrm{B} \mathrm{B}_{1}, \mathrm{CC}_{5}, \mathrm{AA}_{6} & , & , & \mathrm{~B}_{7} \text {. } \\ \mathrm{C} \mathrm{C}_{1}, \mathrm{~A} \mathrm{~A}_{5}, \mathrm{BB}_{6} & , & , & \mathrm{C}_{7} \text {. }\end{array}$

Theorem XVIII.

$\mathrm{AA}_{2}, \mathrm{~B} \mathrm{~B}_{6}, \mathrm{C} \mathrm{C}_{5}$ meet in a point, $\mathrm{A}_{8}$.

$\mathrm{B} \mathrm{B}_{2}, \mathrm{CC}_{6}, \mathrm{AA}_{5} \quad, \quad, \quad \mathrm{~B}_{8}$

THEOREM XIX.

VOL. XXIV. PART I.

$A_{7} A_{8}, B_{7} B_{8}, C_{7} C_{8}$ meet in a point $P_{5}$. 
Theorem XX.

$\mathrm{BC}, \mathrm{B}_{7} \mathrm{C}_{7}, \mathrm{~B}_{8} \mathrm{C}_{8}$ meet in a point, $\boldsymbol{A}_{9}$.

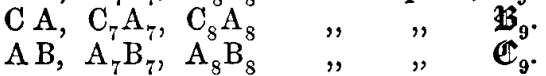

Theorem XXI.

$\mathbb{A}_{9} \mathbf{B}_{9} \mathfrak{C}_{9}$ is a straight line.

Theorem XXII.

B C, A $A_{2}, \quad A_{8} \mathrm{P}_{1}, A_{7} \mathrm{P}_{2}$ all meet in a point, $\mathfrak{A}_{10}$.

$\mathrm{CA}, \quad \mathrm{B} \mathrm{B}, \quad \mathrm{B}_{8} \mathrm{P}_{1}, \mathrm{~B}_{7} \mathrm{P}_{2}{ }^{2}, \cdots, ", \quad \mathfrak{B}_{10^{\circ}}$

$\mathrm{A} \mathrm{B}, \quad \mathrm{C} \mathrm{C}_{2}, \mathrm{C}_{8} \mathrm{P}_{1}, \mathrm{C}_{7}^{7} \mathrm{P}_{2} \quad ", \quad " \quad \mathbb{C}_{10^{\circ}}^{10^{\circ}}$

THEOREM XXIII.

$A \mathfrak{A}_{9}, \mathrm{~A} \mathrm{C}, \mathrm{A} \mathfrak{A}_{10}, \mathrm{~A} \mathrm{~B}$ is a harmonic pencil.

$\mathrm{B} \mathfrak{\mathfrak { B }}_{9}, \mathrm{~B} \mathrm{~A}, \mathrm{~B} \mathfrak{i}_{10}, \mathrm{~B} \mathrm{C}$

$\mathrm{C} \mathfrak{C}_{9}^{9}, \mathrm{CB}, \mathrm{C} \mathfrak{C}_{10}, \mathrm{CA} \quad "$,

TheORE4 XXIV.

$\mathrm{B} \mathrm{C}, \mathrm{A}_{1} \mathrm{P}_{2}, \mathrm{~A}_{2} \mathrm{P}_{1}$ meet in a point, $\mathfrak{A}_{11}$.

$\mathrm{CA}, \mathrm{B}_{1} \mathrm{P}_{2}, \mathrm{~B}_{2} \mathrm{P}_{1} \quad, \quad " \quad \mathfrak{B}_{11}$.

$\mathrm{AB}, \mathrm{C}_{1} \mathrm{P}_{2}, \mathrm{C}_{2} \mathrm{P}_{1} \quad$ ",,$" \quad \mathbb{C}_{11}^{11}$.

Theorem XXV.

$\mathrm{A} \mathfrak{A}_{11}, \mathrm{~B} \mathfrak{B}_{11}, \mathrm{C} \mathfrak{C}_{11}$ intersect in a point, $\mathrm{P}_{6}$.

Theorem XXVI.

$\mathrm{BC}, \mathrm{A}_{1} \mathrm{~A}_{8}, \mathrm{~A}_{2} \mathrm{~A}_{7}$ meet in a point, $\mathfrak{A}_{12}$.

$\mathrm{CA}, \mathrm{B}_{1} \mathrm{~B}_{8}, \mathrm{~B}_{2} \mathrm{~B}_{7} \quad, \quad, \quad \mathfrak{B}_{12}$.

$\mathrm{AB}, \mathrm{C}_{1} \mathrm{C}_{8}^{8}, \mathrm{C}_{2} \mathrm{C}_{7}^{7} \quad " \quad, \quad, \quad \mathbb{C}_{12}^{12^{\circ}}$

Theorem XXVII.

$\mathrm{A} \mathfrak{\mathfrak { A }}_{12}, \mathrm{~B} \mathfrak{z}_{12}, \quad \mathbf{C} \mathfrak{C}_{12}$ intersect in a point, $\mathbf{Q}_{1}$.

Theorear XXVIII.

Let $\mathrm{BC}, \mathrm{A}_{7} \mathrm{~A}_{8}$ meet in $\mathfrak{A}_{13}$

$\begin{array}{llll}\mathrm{BA}, \mathrm{B}_{7} \mathrm{~B}_{8} & , & \mathfrak{B}_{13} \\ \mathrm{AB}, \mathrm{C}_{7} \mathrm{C}_{8} & , & \mathfrak{C}_{13}^{13}\end{array}$

$\left.\begin{array}{l}\mathfrak{A}_{13} \\ \mathfrak{G}_{13} \\ \mathfrak{C}_{13}^{\mathfrak{c}}\end{array}\right\}$;一Then, $\mathrm{A} \mathfrak{A}_{13}, \mathrm{~B} \mathfrak{B}_{13}, \mathbf{C} \mathfrak{C}_{13}$ intersect in a point, $\mathbf{Q}_{2}$.

THEOREM XXIX.

Let $\mathrm{B}_{5} \mathrm{~B}_{6}, \mathrm{C}_{5} \mathrm{C}_{6}$ meet in $\mathrm{A}_{9}$ $\left.\begin{array}{rll}C_{5}^{5} C_{6}, A_{5} A_{6} & \# & B_{9} \\ A_{5} A_{6}, B_{5} B_{6} & \# & C_{9}\end{array}\right\}$; - Then, $A_{9}, \mathrm{~B} \mathrm{~B}_{9}, \mathrm{C} \mathrm{C}_{9}$ intersect in a point, $Q_{3}$.

Theorem XXX.

Let $A_{1} A_{2}, A_{5} A_{6}$ meet in $A_{10}$

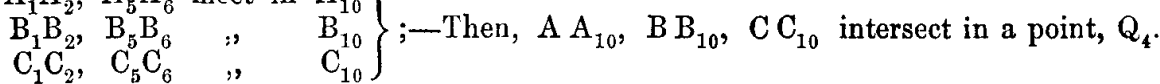




\section{THEOREM XXXI.}

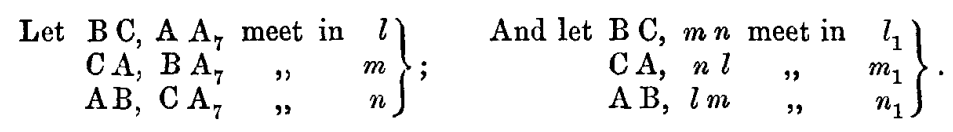

Then $l_{1} m_{1} n_{1}$ is a straight line, as is well known. As it depends entirely on the position of $A_{7}$ let it be called $\phi\left(A_{\eta}\right)$. Then ;-

$\phi\left(A_{7}\right)$ is the polar of $A_{8}$ to the imaginary conic, $\alpha^{2}+\beta^{2}+\gamma^{2}=0$.

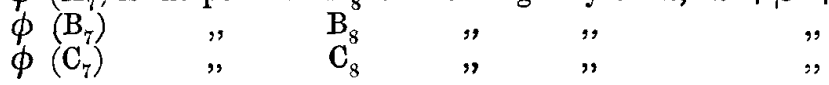

TheoreM XXXII.

$\phi\left(A_{8}\right)$ is the polar of $A_{7}$ to the imaginary conic, $\alpha^{2}+\beta^{2}+\gamma^{2}=0$.



ThEOREM XXXIII.

$\phi\left(\mathrm{P}_{1}\right)$ is the polar of $\mathrm{P}_{2}$ to the imaginary conic, $\alpha^{2}+\beta^{2}+\gamma^{2}=0$.

\section{TheOREM XXXIV.}

$\phi^{-1}\left(\mathfrak{A}_{1} \mathfrak{A}_{1} \mathfrak{C}_{1}\right)$ is the pole of $\mathfrak{A}_{6} \mathfrak{b}_{6} \mathfrak{C}_{6}$ to the conic, $\alpha^{2}+\beta^{2}+\gamma^{2}=0$.

$\phi^{-1}\left(\mathfrak{A}_{6}^{1} \mathfrak{B}_{6} \mathfrak{C}_{6}^{1}\right) \quad, \quad \mathfrak{A}_{1}^{6} \mathfrak{B}_{1}^{6} \mathfrak{C}_{1}^{6}, "$,

\section{Theorem XXXV.}

$\phi^{-1}\left(\mathfrak{A}_{7} \mathfrak{B}_{7} \mathfrak{C}_{7}\right)$ is the pole of $\mathfrak{A}_{8} \mathbb{B}_{8} \mathfrak{C}_{8}$ to the conic, $\alpha^{2}+\beta^{2}+\gamma^{2}=0$.

$\phi^{-1}\left(\mathfrak{A}_{8}^{7} \mathfrak{B}_{8} \mathfrak{C}_{8}^{7}\right), "$ is the pole of $\mathfrak{A}_{7} \mathfrak{A}_{7} \mathfrak{C}_{7}^{8}$

The principle of reciprocation would introduce here a number of Propositions which it is unnecessary to enunciate.

Theorem XXXVI.
Let $\mathrm{A} \mathrm{A}_{1}, \mathrm{~B}_{7} \mathrm{C}_{7}$ meet in $l_{2}$
$\left.\begin{array}{lll}\mathrm{B} \mathrm{B}_{1}, \mathrm{C}_{7} \mathrm{~A}_{7} & \quad & m_{2} \\ \mathrm{CC}_{1}, & \mathrm{~A}_{7} \mathrm{~B}_{7}\end{array}\right\}$



Then $;-\alpha_{1} \beta_{1} \gamma_{1}$ is a straight line.

\section{THEOREM XXXVII.}

Let $\left.\begin{array}{ccc}\mathrm{A} \mathrm{A}_{2}, \mathrm{~B}_{8} \mathrm{C}_{8} & \text { meet in } & l_{3} \\ \mathrm{~B} \mathrm{~B}_{2}, \mathrm{C}_{8} \mathrm{~A}_{8} & , & m_{3} \\ \mathrm{CC}_{2}, \mathrm{~A}_{8} \mathrm{~B}_{8} \quad, & n_{3}\end{array}\right\}$;

And let B C, $m_{3} n_{3}$ meet in $\alpha_{2}$

$$
\left.\mathrm{C} \mathrm{C}_{2}, \mathrm{~A}_{8} \mathrm{~B}_{8} \quad, \quad n_{3}\right\}
$$

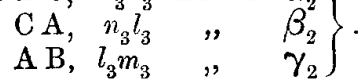

Then; $-\alpha_{2} \beta_{2} \gamma_{2}$ is a straight line.

\section{Theoren XXXVIII.}

Let $\beta_{1} \gamma_{2}, \beta_{2} \gamma_{1}$ meet in $\alpha_{3}$

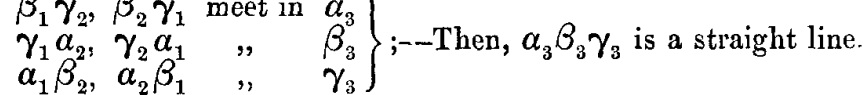




\section{Theorem XXXIX.}

Let $a_{1} \beta_{2}, \gamma_{1} \alpha_{2}$ meet in $\alpha_{4}$

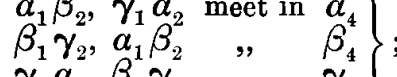

And let $\alpha_{2} \beta_{1}, \gamma_{2} \alpha_{1}$ meet in $\alpha_{5}$ $\left.\gamma_{1} \alpha_{2}, \beta_{1} \gamma_{2} \quad, \quad \gamma_{4}\right\}$



Then ;--

$$
\text { A } \alpha_{4} \alpha_{5} \text { is a straight line. }
$$

$$
\begin{array}{ll}
\mathrm{B} \beta_{4}^{4} \beta_{5} & \quad \% \quad \% \\
\mathrm{C} \gamma_{4} \gamma_{5} \quad " \quad \%
\end{array}
$$

Theorem XL.

A $a_{4} \alpha_{5}, \mathrm{~B} \beta_{4} \beta_{5}, \mathrm{C} \gamma_{4} \gamma_{5}$ meet in a point.

\section{ThEOREM XLI.}

Postulating again, similarly as in Theorems XXXVI. and XXXVII.,-

Let $\mathrm{A} \mathrm{A}_{2}, \mathrm{~B}_{7} \mathrm{C}_{7}$ meet in $\bar{l}_{2}$; and similarly

Also $\mathrm{A} \mathrm{A}_{1}, \mathrm{~B}_{8} \mathrm{C}_{8} \quad, \quad \bar{l}_{3}$; and similarly $\}$. Then;$\bar{\alpha}_{1} \bar{\beta}_{1} \bar{\gamma}_{1}$ is a straight line.

THEOREM XLII.

$\bar{\alpha}_{2} \bar{\beta}_{2} \bar{\gamma}_{2}$ is a straight line.

\section{THeorem XLIII.}

$\bar{\alpha}_{3} \bar{\beta}_{3} \bar{\gamma}_{3}$ is a straight line.

\section{Theorem XLIV.}

A $\bar{\alpha}_{4} \bar{\alpha}_{5}$ is a straight line.

$\mathrm{B} \bar{\beta}_{4} \bar{\beta}_{\overline{5}} \quad, \quad$,

$\mathrm{C} \bar{\gamma}_{4} \bar{\gamma}_{5} \quad, \quad$,

TheOREM XLV.

$\mathrm{A} \bar{\alpha}_{4} \bar{\alpha}_{5}, \mathrm{~B} \bar{\beta}_{4} \bar{\beta}_{5}, \mathrm{C} \bar{\gamma}_{4} \bar{\gamma}_{5}$ meet in a point.

\section{Theorem XLVI.}

Let $\alpha_{1} \alpha_{2}, \bar{\alpha}_{1} \bar{\alpha}_{2}$ meet in $\alpha_{6}$

$$
\begin{aligned}
& \beta_{1} \beta_{2}, \bar{\beta}_{1} \bar{\beta}_{2} \quad, \quad \beta_{6} \quad \text { Then ;- } \\
& \gamma_{1} \gamma_{2}, \bar{\gamma}_{1} \bar{\gamma}_{2},, \quad \gamma_{6}
\end{aligned}
$$

Let $P_{3} Q_{3}$ and $P_{6} Q_{1}$ meet in $Q_{5}$; and let $P_{5} Q_{5}$ and $P_{4} Q_{4}$ meet in $Q_{6}$.

Then the points $P_{1}, P_{2}, P_{3}, P_{4}, P_{5}, P_{6} ; Q_{1}, Q_{2}, Q_{3}, Q_{4}, Q_{5}, Q_{6}$ have very remarkable relations.

Theorem XLVII.

$\mathrm{P}_{1}, \mathrm{P}_{2}, \mathrm{P}_{3}, \mathrm{P}_{4}, \mathrm{P}_{5}, \mathrm{P}_{6}$ all range in a straight line. 


\section{Theorem XLVIII.}

$Q_{1} Q_{4}$ and $Q_{3} Q_{5}$ intersect in $P_{3}$.

Theorem XLIX.

$Q_{2} Q_{5}$ and $Q_{4} Q_{6}$ intersect in $P_{4}$.

Theorem L.

$Q_{1} Q_{3}$ and $P_{1} P_{2}$ intersect in $P_{5}$.

Theorem LI.

$Q_{1} Q_{5}$ and $Q_{3} Q_{4}$ intersect in $P_{6}$.

Theorem LII.

$P_{4} Q_{5}$ and $P_{5} Q_{4}$ intersect in $Q_{2}$.

Theorem LIII.

$P_{1} P_{2}, Q_{1} Q_{2}, Q_{3} Q_{6}$ intersect in a point.

Theorem LIV.

$Q_{1} Q_{3}, Q_{4} Q_{5}, Q_{2} Q_{6}$ intersect in a point,-say

Theorem LV.


$\mathrm{S}_{1} \mathrm{~s}_{2}$ is a straight line.

Theorem LVI.

$\mathrm{S}_{3} \mathrm{~S}_{4}$ is a straight line.

\section{Theorem LVII.}

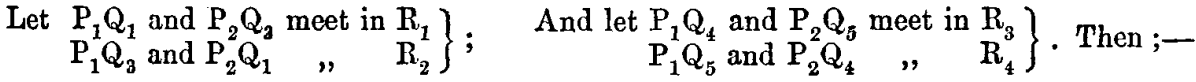
$R_{1} R_{2} P_{\text {is a straight line. }}$

\section{Theorem LVIII.}

$\mathrm{R}_{3} \mathrm{R}_{4}$ is a straight line. 
THEOREM LIX.

$$
\begin{aligned}
& \text { Let } \left.\begin{array}{l}
\mathrm{P}_{1} \mathrm{Q}_{6} \text { and } \mathrm{P}_{2} \mathrm{Q}_{2} \text { meet in } \mathrm{U}_{1} \\
\mathrm{P}_{1} \mathrm{Q}_{2} \text { and } \mathrm{P}_{2} \mathrm{Q}_{6} \quad " \quad \mathrm{U}_{2}
\end{array}\right\} \text { Then;- } \\
& \mathrm{U}_{1} \mathrm{U}_{2} \mathrm{P}_{\text {is a straight line. }} \text {. }
\end{aligned}
$$

Theorem LX. $\mathrm{U}_{1} \mathrm{U}_{2} ; \mathrm{R}_{1} \mathrm{R}_{2}, \mathrm{R}_{3} \mathrm{R}_{4} ; \mathrm{S}_{1} \mathrm{~S}_{2}, \mathrm{~S}_{8} \mathrm{~S}_{4} ; \mathrm{Q}_{1} \mathrm{Q}_{3}, \mathrm{Q}_{4} \mathrm{Q}_{5}, \mathrm{Q}_{2} \mathrm{Q}_{6} ;$ all intersect in $\mathbb{P}$.

\section{Theorem LXI.}

is the pole of $\mathrm{P}_{1} \mathrm{P}_{2} \mathrm{P}_{3} \mathrm{P}_{4} \mathrm{P}_{5} \mathrm{P}_{6}$ to the imaginary conic, $\alpha^{2}+\beta^{2}+\gamma^{2}=0$.

\section{TheOREM LXII.}

The lines $\mathrm{AA}_{1}, \mathrm{BB}_{1}, \mathrm{CC}_{1} ; \mathrm{AA}_{2}, \mathrm{BB}_{2}, \mathrm{CC}_{2}$ cut the sides of the triangle $\mathrm{ABC}$ in six points which lie in the conic;-

$$
a^{2}+\beta^{2}+\gamma^{2}-\left(\frac{g}{h}+\frac{h}{g}\right) \beta \gamma-\left(\frac{h}{f}+\frac{f}{h}\right) \gamma \alpha-\left(\frac{f}{g}+\frac{g}{f}\right) \alpha \beta=0 .
$$

For the six points are inverse or reciprocal points. Substituting the co-ordinates of five of them (the third of the second set being omitted) in the general equation of the second degree, and eliminating the arbitrary constants, gives the conic as above. To substitute the co-ordinates of five of the points, omitting now the third of the first set, amounts evidently to inverting the separate terms in the constants of the above equation; and as this leaves it unaltered, the proof of the theorem is obvious.

\section{Theorem LXIII.}

The lines $\mathrm{AA}_{5}, \mathrm{BB}_{5}, \mathrm{CC}_{5} ; \mathrm{AA}_{6}, \mathrm{BB}_{6}, \mathrm{CC}_{6}$ cut the sides of the triangle $\mathrm{ABC}$ in six points which lie in the conic;-

$$
\alpha^{2}+\beta^{2}+\gamma^{2}-\left(g^{h}+\frac{1}{g h}\right) \beta \gamma-\left(h f+\frac{1}{h f}\right) \gamma \alpha-\left(f g+\frac{1}{f g}\right) a \beta=0 .
$$

\section{THEOREM LXIV.}

The points $\mathfrak{A}_{1} \mathfrak{B}_{1} \mathfrak{C}_{1} ; \mathfrak{A}_{2} \mathfrak{b}_{2} \mathfrak{C}_{2}$ lie in the conic;-

$$
\alpha^{2}+\beta^{2}+\gamma^{2}+\left(f+\frac{1}{f}\right) \beta \gamma+\left(g+\frac{1}{g}\right) \gamma \alpha+\left(h+\frac{1}{h}\right) \alpha \beta=0 .
$$

\section{Theorem LXV.}

If the point $\mathrm{P}_{1}$ move in a straight line, the line $\phi\left(\mathrm{P}_{1}\right)$ will always touch a conic which touches the three sides of the triangle $\mathrm{ABC}$. 
Let the equation of the line in which $\mathrm{P}_{1}$ moves be $l \alpha+m \beta+n \gamma=0$; or substituting the co-ordinates of $P_{1}$ now supposed variable (see Theorem 1),

$$
\phi\left(\mathrm{P}_{1}\right) \text { is } \begin{array}{llll}
\frac{l}{f}+\frac{m}{g}+\frac{n}{h}=0 & . & . & .
\end{array} .
$$

Now $h$ may be considered as constant, since it is the ratios only that are concerned. Take $f$ as the independent variable, then $g$ varies with $f$ by (1); and by the theory of envelopes we have

$$
\begin{aligned}
& \frac{d u}{d f}=0=\left(\frac{d u}{d f}\right)+\left(\frac{d u}{d g}\right) \cdot \frac{d g}{d f} \\
& \pm(l \alpha)^{\frac{1}{2}} \pm(m \beta)^{\frac{2}{2}} \pm(n \gamma)^{\frac{1}{2}}=0 ;-
\end{aligned}
$$

Elimination gives

the equation of a conic touching the sides of the triangle of reference.-Q. E. D.

\section{Theorem LXVI.}

Let lines be drawn from the angles of a triangle through any two points and terminating in the opposite sides ; by joining the extremities of each set of lines so drawn, two other triangles will be formed. The three lines joining the intersections of corresponding sides of these two triangles with the corresponding angle of the original triangle meet in a point.

If the co-ordinates of the two assumed points be $a_{1} b_{1} c_{1}$ and $a_{2} b_{2} c_{2}$ those of the third point are

$$
a_{1} a_{2}\left|\begin{array}{c}
b_{1}, b_{2} \\
c_{1}, c_{2}
\end{array}\right|, \quad b_{1} b_{2}\left|\begin{array}{c}
c_{1}, c_{2} \\
a_{1}, a_{2}
\end{array}\right|, \quad c_{1} c_{2}\left|\begin{array}{c}
a_{1}, a_{2} \\
b_{1}, b_{2}
\end{array}\right| .
$$

Let this point be called the anapole of the two assumed points.

\section{THeorem LXVII.}

The anapoles of $A_{7}, A_{8}$; of $B_{7}, B_{8} ;$ and of $C_{7}, C_{8}$ are in a straight line,-say $\mathbb{A}_{1} B_{1} \mathbb{C}_{1}$

\section{Theorem LXVIII.}

The straight line joining the anapoles of $\mathrm{P}_{1}, \mathrm{~A}_{8}$ and $\mathrm{P}_{2}, \mathrm{~A}_{7}$ passes through $\mathrm{A}$; cutting $\mathrm{BC}$ (say) in $\mathbb{A}_{2}$.


$\mathbb{A}_{2} \mathbb{B}_{2} \mathfrak{C}_{2}$ is a straight line, identical with $\mathfrak{A}_{5} \mathfrak{B}_{5} \mathfrak{C}_{5}$ of THEOREM XII.

\section{Theorem LXIX.}

The lines $A_{1} B_{1} \mathbb{C}_{1}, A_{2} B_{2} C_{2}$ intersect in the anapole of $P_{1}, P_{2}$, which is also the pole of the line $P_{1} P_{2}$ to the imaginary conic, $a^{2}+\beta^{2}+\gamma^{2}=0$. So that the 
anapole of inverse points is the pole of the line joining them-to the same imaginary conic.

\section{THeorem LXX.}

If one of two points remain fixed and the other move in any manner whatever, the locus of the anapoles is a straight line.

THEOREM LXXI.

If two points move away from each other along a straight line with uniform velocities, their anapole will describe a conic section; and if the uniform velocities be equal, a straight line.

\section{Theorem LXXII.}

The anapole of any two points in a conic section passing through the angles of the triangle of reference is invariable, and its co-ordinates are proportional to the sides of the triangle. 